\title{
Vitamin C Blocks Inflammatory Platelet-activating Factor Mimetics Created by Cigarette Smoking
}

\author{
Hans-Anton Lehr, ${ }^{\star}$ Andrew S. Weyrich, ${ }^{\ddagger}$ Rainer K. Saetzler, ${ }^{\S}$ Andrzej Jurek, ${ }^{\ddagger}$ Karl E. Arfors,, Guy A. Zimmerman, ${ }^{\ddagger}$ \\ Stephen M. Prescott," and Thomas M. Mclntyre ${ }^{\ddagger \star \star}$ \\ *Institute for Pathology, Johannes Gutenberg University, Mainz, Germany; ${ }^{\ddagger}$ Department of Medicine, Nora Eccles Harrison CVRTI, \\ University of Utah; ${ }^{\S}$ Department of Physiology, Temple University, Philadelphia, Pennsylvania 19122; "Experimental Medicine Inc., \\ Princeton, New Jersey 08540; "Department of Medicine, Huntsman Cancer Institute, University of Utah; and **Department of \\ Experimental Pathology, University of Utah, Salt Lake City, Utah 84112
}

\begin{abstract}
Cigarette smoking within minutes induces leukocyte adhesion to the vascular wall and formation of intravascular leukocyte-platelet aggregates. We find this is inhibited by platelet-activating factor (PAF) receptor antagonists, and correlates with the accumulation of PAF-like mediators in the blood of cigarette smoke-exposed hamsters. These mediators were PAF-like lipids, formed by nonenzymatic oxidative modification of existing phospholipids, that were distinct from biosynthetic PAF. These PAF-like lipids induced isolated human monocytes and platelets to aggregate, which greatly increased their secretion of IL-8 and macrophage inflammatory protein-1 $\alpha$. Both events were blocked by a PAF receptor antagonist. Similarly, blocking the PAF receptor in vivo blocked smoke-induced leukocyte aggregation and pavementing along the vascular wall. Dietary supplementation with the antioxidant vitamin $\mathrm{C}$ prevented the accumulation of PAF-like lipids, and it prevented cigarette smoke-induced leukocyte adhesion to the vascular wall and formation of leukocyte-platelet aggregates. This is the first in vivo demonstration of inflammatory phospholipid oxidation products and it suggests a molecular mechanism coupling cigarette smoke with rapid inflammatory changes. Inhibition of PAF-like lipid formation and their intravascular sequela by vitamin $\mathrm{C}$ suggests a simple dietary means to reduce smoking-related cardiovascular disease. (J. Clin. Invest. 1997. 99: 2358-2364.) Key words: leukocytes - monocytes • blood platelets $\bullet$ endothelium $\bullet$ chemokines
\end{abstract}

\section{Introduction}

Cigarette smoking has been recognized as a primary risk factor for cardiovascular diseases, the major cause of morbidity and mortality in industrialized nations. Although the mechanisms by which cigarette smoke contributes to cardiovascular disease are not entirely understood, it has been demonstrated that cigarette smoke and its extracts induce diverse inflammatory

Address correspondence to Thomas M. McIntyre, CVRTI, Building 500, University of Utah, Salt Lake City, UT 84112. Phone: 801-5818183; FAX: 801-581-3128; E-mail: mcintyre@cvrti.utah.edu

Received for publication 10 January 1997 and accepted in revised form 14 March 1997.

J. Clin. Invest.

(C) The American Society for Clinical Investigation, Inc. 0021-9738/97/05/2358/07 \$2.00

Volume 99, Number 10, May 1997, 2358-2364 events whose cumulative effects could contribute to cigarette smoke-related pathology. For example, cigarette smoke elicits the recruitment and adhesion of circulating leukocytes to the vessel wall (1-3), the initial step in inflammation $(4,5)$, emphysema (6), and atherosclerosis (7). Recruitment of leukocytes not only into the pulmonary microcirculation $(6,8,9)$, but also into peripheral vascular beds such as the aorta and striated muscle $(1,2)$, suggests that cigarette smoke elicits the formation of circulating inflammatory mediators. Indeed, monocytes isolated just after smoking are in an activated state (10) and plasma isolated just after smoking activates platelets isolated from nonsmokers (11). Cigarette smoke could induce the inflammatory response through a direct effect on target cells as cigarette smoke extracts induce expression of adhesion molecules on endothelial cells $(3,12,13)$. Additional secondary effects of cigarette smoke may extend this response as suggested by the release of inflammatory lipid mediators from cells exposed to smoke extract (14). In fact, material that behaves as platelet-activating factor (PAF), ${ }^{1}$ a potent inflammatory phospholipid (15), is present in blood just after smoking (16).

Cigarette smoke introduces a high burden of radicals into the organism $(17,18)$ and also stimulates the generation of further radicals and reactive oxygen species from activated leukocytes, many of which are chemotactically attracted and sequestered in the lungs $(6,9,13)$. The increased burden of reactive species in the plasma of smokers results in the consumption of the antioxidant vitamin C (19-21), and subsequently the accumulation of various lipid oxidation products in their bloodstream (22-24). We and others (25) have previously shown that oxidation of synthetic (26), cellular (27), and lipoprotein (28) phosphatidylcholine all create a similar spectrum of phospholipid products that activate inflammatory cells through the PAF receptor. These differ from PAF in two ways: they have a variable $s n-2$ residue instead of the acetyl residue of biosynthetic PAF, and they are generated by uncontrolled chemical attack compared with the highly regulated enzymatic synthesis of PAF. Since the oxidative reactions that create these PAFlike lipids have all been performed in a laboratory setting, it is not known whether sufficiently oxidizing conditions exist in vivo to create these inflammatory PAF mimetics. It also is not known whether such mediators could ever accumulate to effective levels in the face of plasma antioxidants and endogenous defense mechanisms such as the constitutively active PAF acetylhydrolase that destroys the biologic activity of PAF and PAF-like lipids (29). Based on these considerations, we asked: $(a)$ whether cigarette smoke could elicit the accumulation of PAF-like lipids in an intact organism; $(b)$ whether these

1. Abbreviations used in this paper: MIP, macrophage inflammatory protein; PAF, platelet-activating factor. 
compounds are the circulating mediators responsible for inflammatory changes observed after cigarette smoke exposure; (c) how these compounds might extend the inflammatory reaction; and $(d)$ whether simple antioxidant protection would suppress their accumulation and thereby suppress the proinflammatory effects of smoking.

\section{Methods}

Lipid isolation and characterization. Plasma was obtained from the EDTA-anticoagulated blood of control animals and those exposed to the smoke of one reference cigarette (1) $15 \mathrm{~min}$ before bleeding. The polar lipids were isolated over an XAD-2 column (Sigma, St. Louis, MO) (30), extracted from the aqueous ethanol (31), and then separated by HPLC on a $5-\mu \mathrm{m} 4.6 \mathrm{~mm} \times 25 \mathrm{~cm}$ ODS column (Rainin Instrument Co., Woburn, MA) using MeOH: $\mathrm{CH}_{3} \mathrm{CN}: \mathrm{H}_{2} \mathrm{O}$ containing $1 \mathrm{mM} \mathrm{NH}_{4}$ acetate (84:15:1) at $1 \mathrm{ml} / \mathrm{min}$ (28) to develop the column. Fractions were collected every minute and $20 \%$ of two adjacent fractions were combined for bioassay. Recovery of $\left[{ }^{3} \mathrm{H}\right] \mathrm{PAF}$ internal standards was $70 \%$ or greater; a separately injected $\left[{ }^{3} \mathrm{H}\right] \mathrm{PAF}$ standard was eluted at $8 \mathrm{~min}$.

Cell isolation and manipulation. Human PMN (26) and peripheral blood monocytes and platelets (32) were isolated from fresh anticoagulated blood as described. The CD18-dependent adhesion of activated PMN was determined (28) by quantifying the number of PMN adherent to a gelatinized surface after a 5 -min incubation at $24^{\circ} \mathrm{C}$. PMN were activated with $10^{-7} \mathrm{M}$ PAF as a positive control, or with HPLC-purified material (20\% of a 2 min fraction) that had been taken to dryness before resuspension in $1 \%$ human serum albumin. WEB 2086 (a gift from Boehringer-Ingelheim, Ridgefield, CT) $(10 \mu \mathrm{M})$ was used to competitively block the PAF receptor as this concentration was sufficient to block the effects of $10^{-7} \mathrm{M}$ PAF in a specific fashion in this assay (26). WEB 2086 binds to the cloned human PAF receptor and competes for PAF and PAF-like lipids obtained from oxidized human low density lipoprotein, and it blocks the ability of PAF and PAFlike lipids to stimulate 293 cells expressing the cloned human PAF receptor (G. Marathe et al., unpublished observations). PAF acetylhydrolase and phospholipase $\mathrm{A}_{2}$ block these effects of PAF and PAF-like lipids, showing phospholipids with a modified $s n-2$ residue are responsible for these activities (not shown). WEB 2086 and WEB 2170 are benzodiazepines that bind the PAF receptor at the same site as PAF (33).

The effect of PAF-like lipids on monocyte-platelet aggregation was determined as follows. Human monocytes $\left(10^{6} \mathrm{cells} / \mathrm{ml}\right)$ were admixed with platelets $\left(10^{8}\right.$ cells $/ \mathrm{ml}$ of M199 [BioWhittaker, Walkersville, $\mathrm{MO}$ ] containing $10 \mu \mathrm{g} / \mathrm{ml}$ polymyxin B [Sigma Chemical Co.]) and incubated or not with WEB $2086(10 \mu \mathrm{m})$ for $30 \mathrm{~min}$. PAF or partially purified PAF-like lipids were then added and, after 5 or $30 \mathrm{~min}$, the cells in an aliquot were recovered onto a glass slide by Cytospin ${ }^{\circledR}$ (Shandon Inc., Sewickley, PA) centrifugation. These were fixed with $4 \%$ formaldehyde for $20 \mathrm{~min}$ and the endogenous alkaline phosphatase activity of platelets was visualized by counterstaining with an alkaline phosphatase substrate kit (Vector Laboratories, Inc., Burlingame, CA). The red reaction product was examined using Nomarski interference contrast optics. For cytokine secretion by monocyteplatelet aggregates, the time of incubation was extended to $18 \mathrm{~h}$, at which time the medium was collected, clarified by centrifugation, and cytokine content determined by ELISA as previously described (32).

Animal model. The dorsal skinfold chamber was implanted in Syrian Golden hamsters maintained ad libitum on standard chow as described (2). Vitamin $\mathrm{C}$ was administered to certain animals by including it in the diet $(10 \mathrm{~g} / \mathrm{kg}$ ). Exposure to cigarette smoke (one 2R1 research cigarette; University of Kentucky, Lexington, KY) was performed as described (1), and achieved plasma nicotine and carboxyhemoglobin levels similar to those present in human smokers. Analysis of leukocyte interactions in the microcirculation (34) before smoke exposure and at the stated times after exposure was performed in four to six arterioles and venules (20-60 $\mu \mathrm{m}$ diameter) per observation window. Leukocytes were visualized after intravascular injection of acridine orange (35) and cells remaining stationary at the vascular wall for $30 \mathrm{~s}$ were classified as adherent. Vessel diameters and red cell velocities were analyzed offline from video recordings as described (34). Leukocyte/platelet aggregates, defined as a minimum of three leukocytes per aggregate, tumbling through microvessels
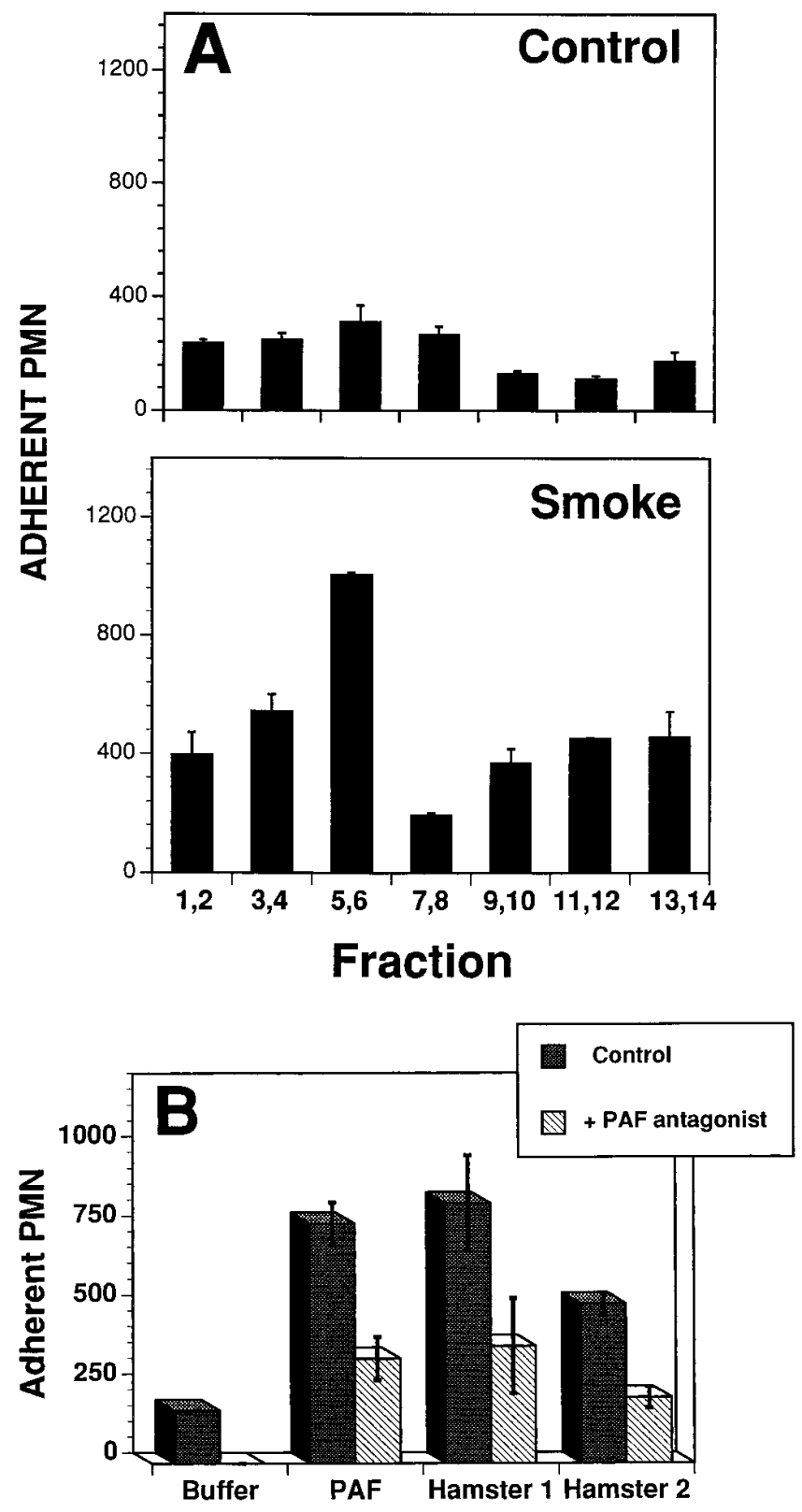

Figure 1. Isolation and characterization of PAF-like lipids from the plasma of cigarette smoke-exposed hamsters. (A) HPLC chromatogram of bioactive lipids. Plasma was obtained from control hamsters or animals exposed 15 minutes earlier to one cigarette (2R1, University of Kentucky research cigarette). The lipids were extracted and the polar lipid fraction was isolated and separated by ODS HPLC before 2-min fractions were assayed for stimulation of PMN adhesion. Separately injected $\left[{ }^{3} \mathrm{H}\right] \mathrm{PAF}$ standards were eluted at $8 \mathrm{~min}$. (B) Effect of PAF receptor blockade. PMN were pretreated with a specific PAF receptor antagonist WEB 2086, and then incubated with $10^{-7} \mathrm{M}$ PAF or lipid fraction 5,6 from the plasma of smoke-exposed animals in an albumin-containing buffer. Adhesion values are the number of PMN per field expressed as median and range of duplicate assays and represent two independent experiments. 
within a 30-s observation period were counted in four to six venules per observation window before and at defined times after smoking. When the effect of a PAF receptor antagonist (WEB 2170) was to be examined, animals were given $1 \mathrm{mg} / \mathrm{kg}$ body wt i.v., $10 \mathrm{~min}$ before smoke exposure (36). Leukocyte-platelet aggregates were isolated from EDTA-anticoagulated blood, fixed, and stained for analysis by electron microscopy as before (37).

\section{Results}

Smoking induces the accumulation of PAF-like lipids. We exposed hamsters to the smoke from one reference cigarette, extracted lipids from their plasma, separated the lipids by HPLC, and examined the individual fractions for their ability to activate leukocytes in vitro. Polar lipids extracted from plasma just 15 min after smoke exposure, but not from control plasma, exerted a marked adhesion-promoting effect on human neutrophils (Fig. $1 A$ ). The stimulatory material did not migrate with the authentic PAF standard, but instead eluted where oxidized phospholipids with PAF-like activity migrate (28). These PAF mimetics differ from PAF in that their $s n-2$ residue is variable (hence the differing chromatographic properties); moreover, they are generated by an uncontrolled free radical attack on phospholipid acyl residues rather than synthesized through a carefully controlled enzymatic pathway (reviewed in reference 38). Considerable amounts of these inflammatory agonists accumulate in the plasma upon exposure to cigarette smoke as their bioactivity was equivalent to $10^{-7} \mathrm{M}$ PAF per ml plasma (Fig. $1 B$ ), a maximally effective concentration of PAF for these cells. To determine whether the lipid fraction isolated from smoke-exposed plasma activated leukocytes via the PAF receptor, we blocked this receptor with a specific competitive antagonist. This antagonist attenuated the response to $10^{-7} \mathrm{M}$ PAF and the fractionated lipid to a comparable extent (Fig. $1 B$ ). Thus, PAF-like lipids, rather than authentic PAF (14, $35)$, are generated in vivo in response to cigarette smoke, consistent with the recent demonstration of in vivo plasma lipid oxidation in smokers $(22,24)$.
PAF-like lipids induce cytokine synthesis by platelet-leukocyte aggregates. We invariably observe the rapid formation of circulating leukocyte-platelet aggregates in hamsters exposed to smoke $(1,2)$, a reaction that can block the microcirculation or act as a nidus for thrombus formation. To test whether the PAF-like lipids extracted from the plasma of smoke-exposed hamsters are also responsible for this aggregate formation, we added the isolated lipid fraction to purified mixtures of human platelets and monocytes. Unstimulated platelet-monocyte mixtures do not aggregate (Fig. $2 A$ ), while the isolated PAF-like lipids induced clustering (Fig. $2 B$ ) that was abolished by pretreatment of the cells with a PAF-receptor antagonist (Fig. 2 $C)$. Similar bridging of leukocytes by activated, dendritic platelets is also found when aggregates isolated from the blood of smoke-exposed hamsters are examined (Fig. $2 D$ ).

One important attribute of monocyte-platelet aggregates that is not shown by individual monocytes (39) is a greatly enhanced ability to synthesize and secrete inflammatory cytokines of both the C-C cytokine family (that attract and activate monocytes; e.g., macrophage inflammatory protein [MIP]-1 $\alpha$ ), and $\mathrm{C}-\mathrm{X}-\mathrm{C}$ cytokine family (that attract and activate neutrophils; e.g., IL-8). This occurs because activated platelets secrete the monocyte agonist RANTES and they express P-selectin that induces a second, outside-in signal in monocytes adhering to activated platelets through this molecule (32). We wished to determine if a similar synergistic activation of monocyte aggregates occurred when platelets and monocytes were activated via their PAF receptor, rather than the RANTES receptor, and whether the PAF mimetics were agonists for this activation. We examined monocyte synthesis of MIP- $1 \alpha$ and IL-8 in response to the PAF-like lipids isolated from smokeexposed hamsters, but found (Fig. 3) that they evoked little synthesis of these cytokines by individual monocytes. However, in the presence of platelets, these PAF-like lipids induced a substantial synthesis of both cytokines by monocyte-platelet aggregates. All of this stimulation could be blocked by treating the platelets and the monocytes with a PAF receptor antagonist (Fig. 3), as well as by inhibiting monocyte-platelet aggrega-
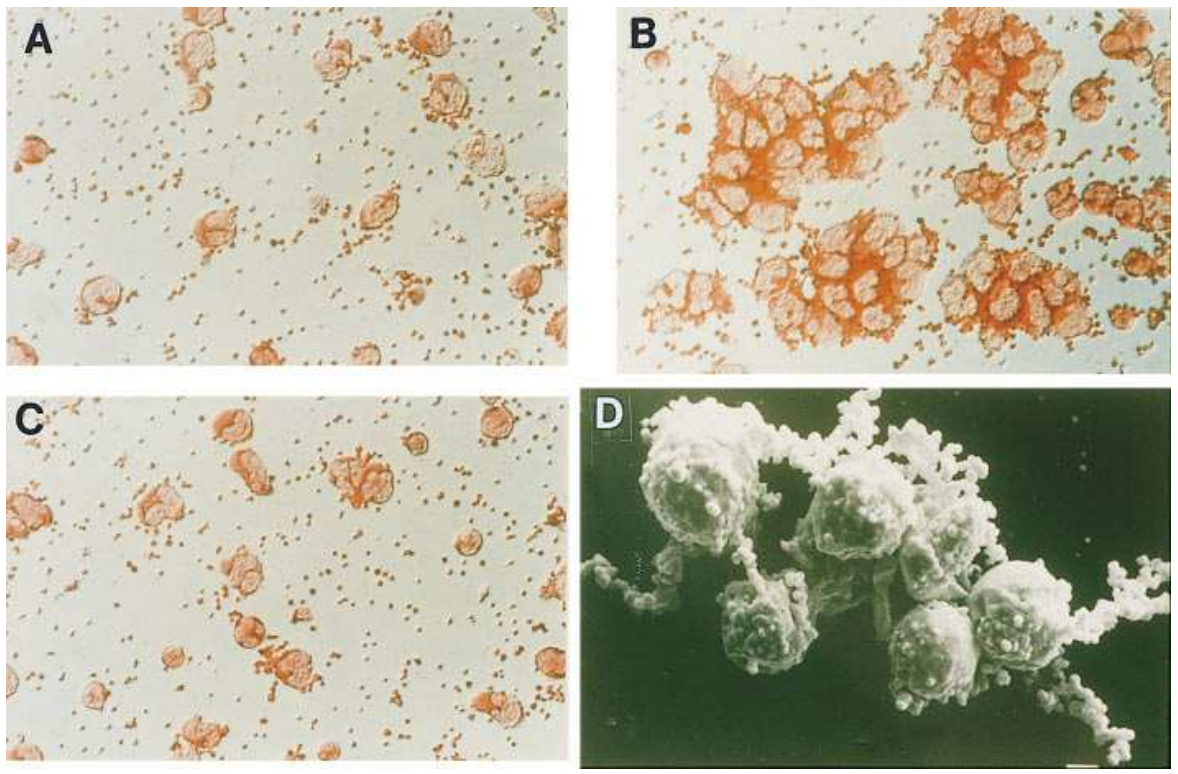

Figure 2. PAF-like lipids extracted from plasma of smoke-exposed hamsters induce monocyte-platelet aggregation via the PAF receptor. Isolated human platelets and monocytes were exposed for $30 \mathrm{~min}$ (a 5-min exposure produced similar changes) to buffer $(A)$, or incubated with HPLC fraction 5,6 from smoke-exposed plasma without $(B)$ or with $(C)$ pretreatment with the PAF-receptor antagonist WEB 2086. These results are typical of three experiments. (D) Scanning electron micrograph of leukocyte aggregates isolated from the blood of a hamster 15 min after smoke exposure. Note the involvement of activated, dendritic platelets forming threadlike bridges between individual leukocytes (bar $=1 \mu \mathrm{m})$. 


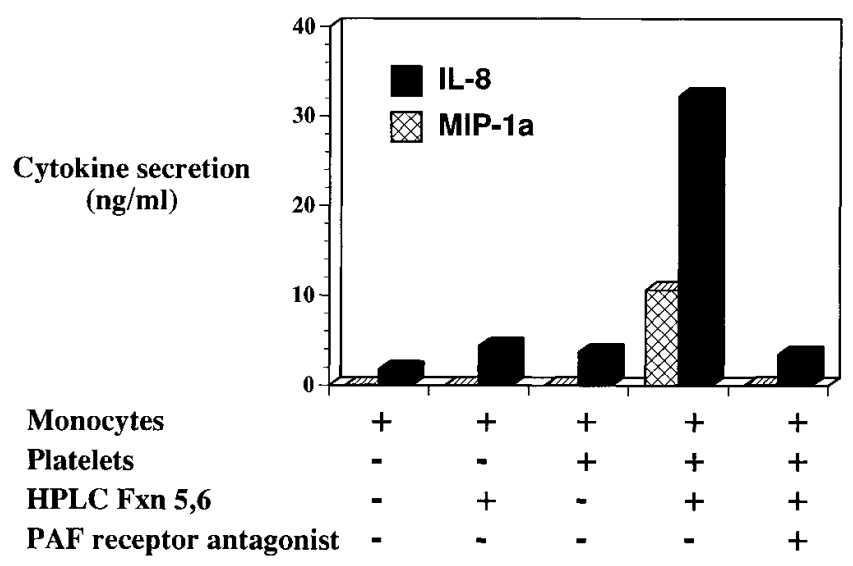

Figure 3. PAF-like lipids induce monocyte-platelet aggregates to secrete inflammatory cytokines. Freshly isolated, unactivated human monocytes were incubated with the stated combination of unactivated human platelets, PAF-like lipid, or WEB 2086. After $18 \mathrm{~h}$, the cells were removed by centrifugation and the supernatants examined for IL- 8 or MIP- $1 \alpha$ secretion by ELISA. Typical of three similar experiments.

tion with a monoclonal antibody to P-selectin (data not shown). These data suggest that platelet-monocyte aggregates may function as circulating factories for inflammatory cytokine synthesis that extend the inflammatory reaction.

PAF-like lipids account for smoking-induced inflammation. We used intravital fluorescence microscopy in a dorsal skinfold chamber preparation to visualize aggregate formation and leukocyte interaction with microvascular endothelium in response to cigarette smoke to understand the potential role of PAF-like lipids in vivo. It is this localization of cells in a distal somatic vascular bed that suggests that circulating inflammatory mediators are generated in response to cigarette smoke. To determine whether these postulated mediators are circulating PAF-like lipids, we blocked the PAF receptor with a specific competitive antagonist. We found that smoke-induced leukocyte adhesion to both arteriolar and venular endothelium was significantly inhibited by pretreating the animals with a highly selective PAF receptor antagonist before smoke exposure (Fig. 4, $A$ and $B$ ). We also found that blocking the PAF receptor significantly inhibited the formation of intravascular leukocyte-platelet aggregates (Fig. 4 C). Taken together, these results show that PAF-like lipids are rapidly formed in vivo in smoke-exposed animals, and that they account for leukocyte adhesive interactions in the microcirculation induced by cigarette smoke.

Vitamin C suppresses PAF-like lipid formation and inflammation. Cigarette smoke contains numerous long-lived free radicals, estimated at $5 \mathrm{nmol}$ per puff (18), in addition to nicotine and a multitude of noxious chemicals. In a previous study (1), we observed that cigarette smoke-induced adhesion was blocked by superoxide dismutase, suggesting that the oxidizing radicals in cigarette smoke account for the rapid inflammatory response in the microcirculation. We now asked whether an antioxidant could prevent the accumulation of PAF-like lipids that initiate the inflammatory reaction induced by cigarette smoke. To test this, we added the antioxidant vitamin $\mathrm{C}$ to the diet of hamsters, and extracted and purified PAF-like lipids
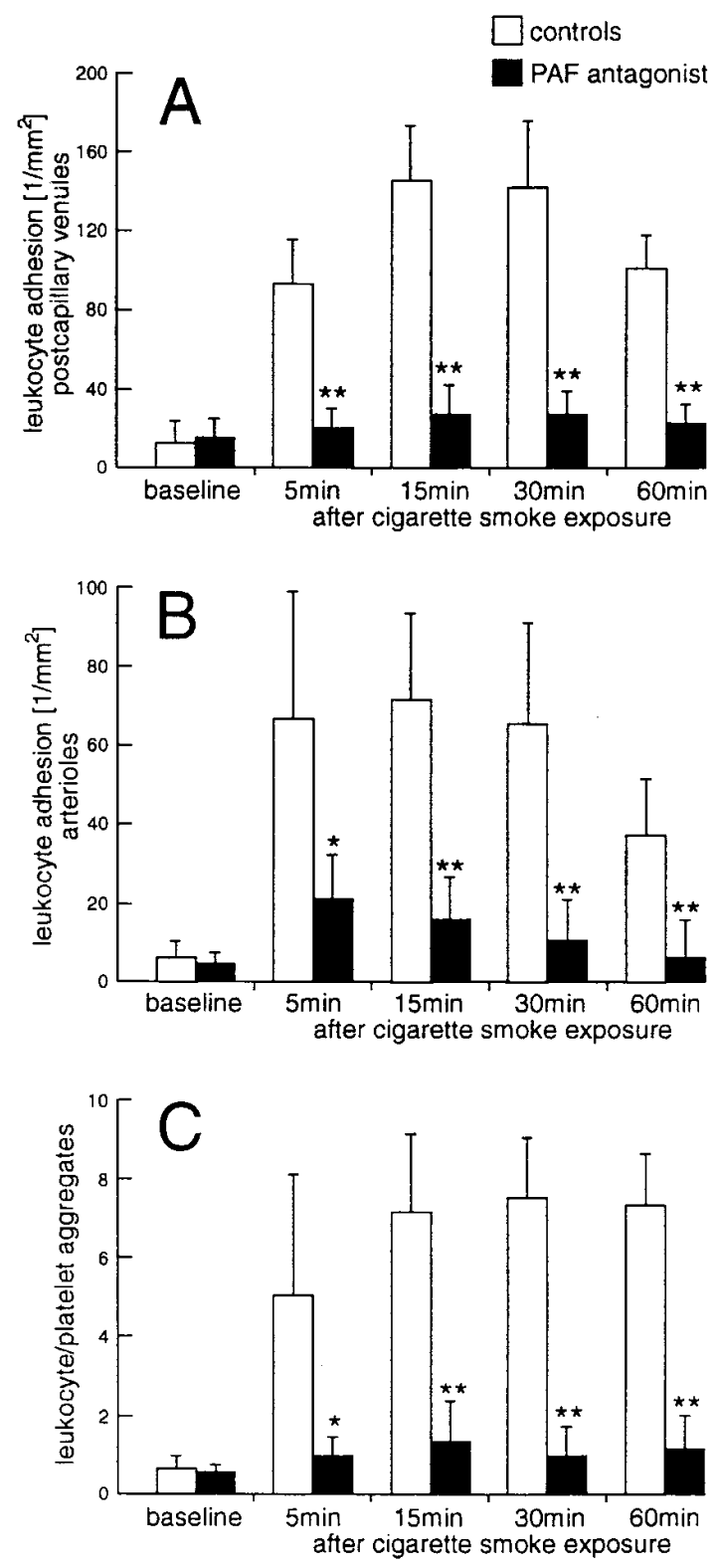

Figure 4. Smoking stimulates in vivo leukocyte adhesion to endothelial cells and leukocyte-platelet aggregate formation via the PAF receptor. Leukocyte adhesion to the endothelium of venules $(A)$ and arterioles $(B)$ was assessed by intravital fluorescence microscopy in the dorsal skinfold chamber preparation in hamsters before (time 0 ) and at defined times after smoke exposure. Adherent leukocytes are expressed per square millimeter of endothelial surface. (C) Effect of a PAF-receptor antagonist on leukocyte aggregate formation. Leukocyte-platelet aggregates (minimum of three leukocytes per aggregate) tumbling through microvessels within a 30-s observation period were counted at defined times after smoke exposure. Measurements were performed in control animals (open bars) and in animals pretreated with the PAF-receptor antagonist WEB 2170 (filled bars) $10 \mathrm{~min}$ before smoke exposure. Data are mean $\pm \mathrm{SD}$ of $n=7$ animals per group. $* P<0.05, * * P<0.01$ vs. comparable values in control animals (Wilcoxon test).

from control and vitamin $\mathrm{C}$-fed animals after smoke exposure. We then compared the amount of stimulatory activity in these extracts to that of a maximally effective concentration of PAF. We observed, with the exception of a single animal, that expo- 


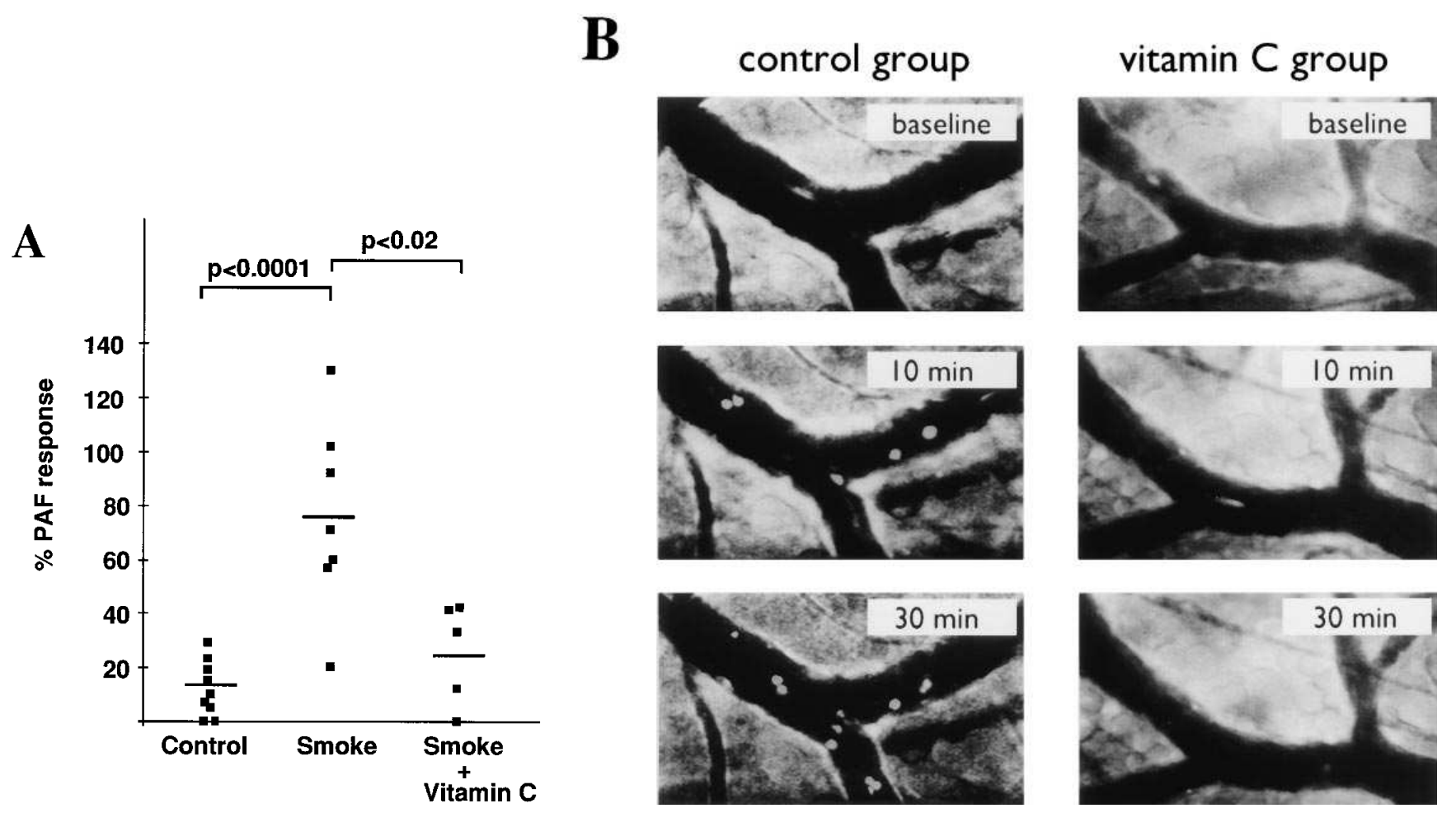

Figure 5. Vitamin C suppresses PAF-like lipid accumulation and leukocyte-endothelium interaction in smoke-exposed hamsters. ( $A$ ) PAF-like lipids were extracted and assayed for biological activity as described in Fig. 1. PMN adhesion induced by 20\% of the HPLC lipid fraction 5,6 was compared with the maximally inducible PAF response for each donor's PMN. Adhesion-promoting activity in plasma from smoke-exposed plasma $(76 \pm 36 \% \mathrm{SD})$ was significantly (two-tailed $t$ test) different from the activity in control plasma ( $12 \pm 10 \%$ SD) and in smoke-exposed plasma obtained from animals fed a vitamin C-supplemented diet for $1 \mathrm{wk}$ before the experiments (26 $\pm 19 \% \mathrm{SD})$. (B) Intravital microscopic evaluation of leukocyte rolling and adhesion. Animals fed vitamin C or not were exposed to cigarette smoke, leukocytes stained with acridine orange by intravascular injection, and visualized by fluorescent microscopy. The white dots are adherent cells, while the elongated streaks are slowly migrating cells.

sure to cigarette smoke results in the accumulation of significant levels of circulating PAF-like lipids, and that vitamin $\mathrm{C}$ prefeeding reduces the amount of circulating PAF-like lipids to background levels (Fig. $5 \mathrm{~A}$ ). While this effect of vitamin $\mathrm{C}$ likely derives from scavenging the radicals $(40,41)$ that initiate phospholipid oxidation to PAF-like lipids (26), an alternative mechanism is that vitamin $\mathrm{C}$ enhances their degradation in the circulation. This is more than a formal alternative since PAF acetylhydrolase, the degradative enzyme that acts on oxidized phospholipids $(29,42)$, is itself susceptible to oxidative inactivation by components of cigarette smoke (43). However, we found no difference in acetylhydrolase activity among the different animal groups (controls, 10.4 \pm 1.3 ; smoke, 10.3 \pm 1.9 ; vitamin $\mathrm{C} / \mathrm{smoke}, 11.1 \pm 1.6 \mu \mathrm{mol} / \mathrm{ml}$ per $\mathrm{h}$ ), so vitamin $\mathrm{C}$ is not able to influence the degradative rate of PAF-like lipids. As in an analogous study (2), dietary vitamin $\mathrm{C}$ also suppresses the systemic inflammatory response that rapidly develops after exposure to cigarette smoke (Fig. $5 \mathrm{~B}$ ). Thus, oxidative formation of PAF-like lipids accounts for the rapid pavementing of leukocytes on the vascular wall and the formation of leukocyte-platelet aggregates in the circulation after cigarette smoking. Vitamin $\mathrm{C}$ and PAF-receptor antagonists are effective at suppressing this inflammation as they both affect the same chain of events; vitamin $\mathrm{C}$ acts at the top of the oxidative cascade to prevent mediator formation, while the PAF receptor antagonist blocks the biologic activity of these oxidative products.

\section{Discussion}

In this study, we demonstrate for the first time that PAF-like lipids are formed and accumulate in vivo, and that biologically relevant levels are achieved after exposure to a single cigarette. In analogy to previous in vitro studies $(25-28,44)$, we propose that these compounds are formed by attack of cigarette smoke-derived radicals or reactive oxygen species on plasma or membrane phospholipids. The oxidative modification of phosphatidylcholine to PAF-like lipids is not due to fragmentation of the $s n$-2 fatty acyl residue to an acetyl residue to create PAF or its acyl analog. Rather, oxidation generates a variety of currently unidentified residues that are also capable of high affinity recognition by the PAF receptor. Despite the unusual nature of the $s n-2$ residues of these modified phospholipids, partial purification shows that they are at least within an order of magnitude as potent as PAF itself (unpublished observations). The observation that these PAF-like lipids are formed in vivo deserves particular attention when taking into consideration the powerful antioxidant capacity of plasma, as well as specific endogenous defense mechanisms such as a constitutively active PAF acetylhydrolase that destroys the bioactivity of PAF and PAF-like lipids (29). Indeed, we found that plasma vitamin $\mathrm{C}$ levels (unpublished observations) and PAF acetylhydrolase (this study) were not affected by this cigarette smoke exposure, suggesting that PAF-like lipids accumulate in 
vivo despite the presence of these powerful defense mechanisms. The realization that compounds other than authentic PAF are capable of stimulating the PAF receptor and occur in vivo suggests that care needs to be applied to the interpretation of experiments in which PAF-receptor antagonists are used to inhibit defined biological responses.

Through the combined use of in vitro and in vivo techniques, we provide evidence in this study that PAF-like lipids found in the plasma of cigarette smoke-exposed hamsters act as the primary mediators of the subsequent microvascular events: first, leukocyte adhesion to endothelial cells as well as leukocyte-platelet aggregation could be observed both in vivo after cigarette smoke exposure (Fig. 4) and in vitro after stimulation with the PAF-like lipids isolated from cigarette smokeexposed hamsters (Figs. 1 and 2). Second, the adhesion- and aggregation-promoting activity was inhibited (in vitro and in vivo to a similar extent) by a synthetic PAF-receptor antagonist (Figs. 1, 2, and 4). Third, the water-soluble antioxidant vitamin C prevented the formation of PAF-like lipids (Fig. 5) and also the cigarette smoke-induced leukocyte adhesion and aggregation (Fig. 5). Our findings in the whole animal and isolated human cells are completely congruent, which shows not only the tight relationship between in vivo and in vitro approaches, but also the usefulness of this animal model to investigate human disease processes. We believe it is particularly important that inhibition of the PAF receptor blocks all the functional changes we examined in the microcirculation, and that vitamin $\mathrm{C}$ blocks accumulation of the PAF-like lipids and these microcirculatory events. This allows us to conclude that it is the oxidative formation of these PAF mimetics that causes leukocyte adhesion to platelets and to the vascular wall.

One of the subsequent events promoted by PAF-like lipids is the selective activation of platelet-monocyte aggregates, as compared with single monocytes, to secrete IL- 8 and MIP- $1 \alpha$ (and monocyte chemotactic protein-1, not shown). In congruence with our previous work $(32,39)$, we find that engagement of the P-selectin glycoprotein ligand-1 (PSGL-1) receptor of monocytes with the P-selectin abundantly displayed on the surface of activated platelets allows monocytes to synthesize and secrete these important inflammatory cytokines. Thus, even though both individual and monocyte-platelet aggregates are exposed to an appropriate agonist, only the multicellular aggregate responds to extend the inflammatory reaction. The in vivo demonstration of circulating monocyte-platelet aggregates (as in Fig. $2 \mathrm{D}$ ) that rapidly accumulate (Fig. $4 C$ ) after exposure of the hamsters to cigarette smoke suggests the potential for localized production of both PMN and monocytic chemoattractants and activators should the aggregates lodge in the microcirculation. In this regard, we note a pathologic complication of cigarette smoking that should be investigated with the formation of hyperexcitable leukocyte aggregates in mind. Thromboangitis obliterans (Buerger's disease) is a segmental occlusive disease of peripheral small and medium sized arteries and veins that is a consequence of heavy cigarette smoking in certain populations (45). The lesions in this disease are inflammatory in nature with an occlusive highly cellular thrombus showing PMN and leukocyte infiltration. We hypothesize that PAF-like lipids generated by smoking create platelet-leukocyte aggregates that become lodged in the microcirculation, and that thrombogenesis at this nidus is stimulated by further exposure to circulating PAF-like lipids that stimulate secretion of both $\mathrm{C}-\mathrm{X}-\mathrm{C}$ and $\mathrm{C}-\mathrm{C}$ chemokines that stimulate leukocyte infiltration. The only current treatment for this disease is smoking cessation, which would stop the exposure to PAF-like lipids and thereby arrest the development and maturation of the occlusive lesions. Our results with vitamin $\mathrm{C}$ suggest a secondary route to the same end point for those patients who are unable to stop smoking.

The ability of the antioxidant vitamin $\mathrm{C}$ to inhibit the generation of PAF-like lipids and the adhesion- and aggregationpromoting action of cigarette smoke in vivo deserves further attention. These effects were introduced by raising vitamin $\mathrm{C}$ plasma levels from $<20 \mathrm{mmol}$ in control hamsters to almost 60 mmol in vitamin C-fed hamsters (37). Based on epidemiological data, the threshold levels for effective protection from cardiovascular disease by vitamin $\mathrm{C}$ has been estimated at $40-50$ mmol (46). This protective level was surpassed by the dietary vitamin $\mathrm{C}$ regime of our study, indicating that the vitamin levels in the animals translate well into data in human subjects. Thus, we found low baseline vitamin levels in control hamsters, corresponding to a predicted high risk of cardiovascular disease, and we found these levels to be high after vitamin $\mathrm{C}$ supplementation, which would correspond to a predicted low risk of cardiovascular disease $(19,20,46)$. The demonstration in the present study that vitamin $\mathrm{C}$ inhibits the cigarette smoke-induced accumulation of PAF-like lipids and the microcirculatory events they cause is in line with previous studies that show vitamin $\mathrm{C}$ suppresses the oxidation of plasma lipoproteins $(23,47)$, and with epidemiological evidence that vitamin C significantly protects against cardiovascular disease (19, $20,46)$. Furthermore, other products, such as the potent vasoconstrictor 8-epi-prostaglandin $\mathrm{F}_{2 \alpha}$, are produced by oxidative attack on cellular and circulating lipids in response to cigarette smoke $(22,24)$, and the formation of these oxidized fatty acids is also suppressed by vitamin $\mathrm{C}$ feeding (24). Formation of vasoconstrictors in conjunction with proinflammatory PAF-like lipids is likely to be a particularly problematic consequence of smoking, so it is important that the generation of both types of mediators is suppressed by dietary vitamin C. Vitamin C supplementation may be particularly efficacious in smokers: they suffer from a steady consumption of vitamin $\mathrm{C}$ and thus have significantly lower plasma levels than nonsmokers $(19,20)$ unless dietary supplementation is started to reach normalized levels (10). All these data suggest that smokers might reduce their cardiovascular risk by adding vitamin C-rich foods or vitamin $\mathrm{C}$ supplements to their daily diet.

\section{Acknowledgments}

We appreciate the aid of Diana Stafforini in determining PAF acetylhydrolase activity, Kurt Albertine for microscopy aid, and Margaret Vogel, Angela Le, and Sue Cowley for leukocyte isolation. We appreciate the assays of PAF-like lipids by Gopal Marathe and Sean Davies, and we thank Patrick Gray (ICOS Corp., Bothell, WA) for rhPAFR-293 cells. We thank Dr. Vijayanand Modur for suggesting a potential connection of this work to Buerger's disease.

This work was supported by the National Institutes of Health (HL-44513, HL-50153, HL-46022) and the Nora Eccles Treadwell Foundation.

\section{References}

1. Lehr, H.A., E. Kress, M.D. Menger, H.P. Friedl, C. Hubner, K.E. Arfors, and K. Messmer. 1993. Cigarette smoke elicits leukocyte adhesion to endothelium in hamsters: inhibition by CuZn-SOD. Free Radical Biol. Med. 14:573-581. 
2. Lehr, H.A., B. Frei, and K.E. Arfors. 1994. Vitamin C prevents cigarette smoke-induced leukocyte aggregation and adhesion to endothelium in vivo. Proc. Natl. Acad. Sci. USA. 91:7688-7692.

3. Kalra, V.K., Y. Ying, K. Deemer, R. Natarajan, J.L. Nadler, and T.D. Coates. 1994. Mechanism of cigarette smoke condensate induced adhesion of human monocytes to cultured endothelial cells. J. Cell Physiol. 160:154-162.

4. Lorant, D.E., M.K. Topham, R.E. Whatley, R.P. McEver, T.M. McIntyre, S.M. Prescott, and G.A. Zimmerman. 1993. Inflammatory roles of P-selectin. J. Clin. Invest. 92:559-570.

5. Ley, K. 1995. Gene-targeted mice in leukocyte adhesion research. Microcirculation (NY). 2:141-150.

6. MacNee, W., B. Wiggs, A.S. Belzberg, and J.C. Hogg. 1989. The effect of cigarette smoking on neutrophil kinetics in human lungs. N. Eng. J. Med. 321: 924-928.

7. Berliner, J.A. and J.W. Heinecke. 1996. The role of oxidized lipoproteins in atherogenesis. Free Radical Biol. Med. 20:707-727.

8. Klut, M.E., C.M. Doerschuk, S.F. Van Eeden, A.R. Burns, and J.C. Hogg. 1993. Activation of neutrophils within pulmonary microvessels of rabbits exposed to cigarette smoke. Am. J. Respir. Cell Mol. Biol. 9:82-89.

9. Bosken, C.H., C.M. Doerschuk, D. English, and J.C. Hogg. 1991. Neutrophil kinetics during active cigarette smoking in rabbits. J. Appl. Physiol. 71: 630-637.

10. Weber, C., W. Erl, K. Weber, and P.C. Weber. 1996. Increased adhesiveness of isolated monocytes to endothelium is prevented by vitamin $\mathrm{C}$ intake in smokers. Circulation. 93:1488-1492.

11. Blache, D. 1995. Involvement of hydrogen and lipid peroxides in acute tobacco smoking-induced platelet hyperactivity. Am. J. Physiol. 268:679-685.

12. Shen, Y., V. Rattan, C. Sultana, and V.K. Kalra. 1996. Cigarette smoke condensate-induced adhesion molecule expression and transendothelial migration of monocytes. Am. J. Physiol. 39:H124-H1633.

13. Kilburn, K.H., and W. McKenzie. 1975. Leukocyte recruitment to airways by cigarette smoke and particle phase in contrast to cytotoxicity of vapor. Science (Wash. DC). 189:634-637.

14. Shoji, S., R.F. Ertl, S. Koyama, R. Robbins, G. Leikauf, S. von Essen, and S.I. Rennard. 1995. Cigarette smoke stimulates release of neutrophil chemotactic activity from cultured bovine bronchial epithelial cells. Clin. Sci. 88:337-344.

15. Whatley, R.E., G.A. Zimmerman, S.M. Prescott, and T.M. McIntyre. 1996. Platelet-activating factor and PAF-like mimetics. In Handbook of Lipid Research, Volume 8: Lipid Second Messengers. R.M. Bell, editor. Plenum Press, New York. 239-276.

16. Imaizumi, T., K. Satoh, H. Yoshida, H. Kawamura, M. Hiramoto, and S. Takamatsu. 1991. Effect of cigarette smoking on the levels of platelet-activating factor-like lipid(s) in plasma lipoproteins. Atherosclerosis. 87:47-55.

17. Church, D.F., and W.A. Pryor. 1985. Free-radical chemistry of cigarette smoke and its toxicological implications. Environ. Health Perspect. 64:111-126.

18. Pryor, W.A., and K. Stone. 1993. Oxidants in cigarette smoke. Ann. NY Acad. Sci. 686:12-28.

19. Riemersma, R.A., D.A. Wood, C.C.A. Macintyre, R.A. Elton, K.F. Gey, and M.F. Oliver. 1991. Risk of angina pectoris and plasma concentrations of vitamins A, C, and E and carotene. Lancet. 337:1-5.

20. Enstrom, J.E., L.E. Kanim, and M.A. Klein. 1992. Vitamin C intake and mortality among a sample of the United States population. Epidemiology. 3: 194-202.

21. Frei, B., L. England, and B.N. Ames. 1989. Ascorbate is an outstanding antioxidant in human blood plasma. Proc. Natl. Acad. Sci. USA. 86:6377-6381.

22. Morrow, J.D., B. Frei, A.W. Longmire, J.M. Gaziano, S.M. Lynch, Y. Shyr, W.E. Strauss, J.A. Oates, and L.J. Roberts. 1995. Increase in circulating products of lipid peroxidation $\left(\mathrm{F}_{2}\right.$-isoprostanes $)$ in smokers. N. Eng. J. Med. 332:1198-1203.

23. Harats, D., M. Ben-Naim, Y. Dabach, D.G. Hollander, E. Havivi, O. Stein, and Y. Stein. 1990. Effect of vitamin C and E supplementation on susceptibility of plasma lipoproteins to peroxidation induced by acute smoking. Atherosclerosis. 85:47-54.

24. Reilly, M., N. Delanty, J.A. Lawson, and G.A. FitzGerald. 1996. Modulation of oxidant stress in vivo in chronic cigarette smokers. Circulation. 94:19-25.

25. Tanaka, T., H. Minamino, S. Unezaki, H. Tsukatani, and A. Tokumura. 1993. Formation of platelet-activating factor-like phospholipids by $\mathrm{Fe}^{2+} / \mathrm{ascor}$ bate/EDTA-induced lipid peroxidation. Biochim. Biophys. Acta. 1166:264-274.

26. Smiley, P.L., K.E. Stremler, S.M. Prescott, G.A. Zimmerman, and T.M. McInytre. 1991. Oxidatively fragmented phosphatidylcholines activate human neutrophils through the receptor for platelet-activating factor. J. Biol. Chem. 266:11104-11110.

27. Patel, K.D., G.A. Zimmerman, S.M. Prescott, and T.M. McIntyre. 1992. Novel leukocyte agonists are released by endothelial cells exposed to peroxide. J. Biol. Chem. 267:15168-15175.

28. Heery, J.M., M. Kozak, D.M. Stafforini, D.A. Jones, G.A. Zimmerman, T.M. McIntyre, and S.M. Prescott. 1995. Oxidatively modified LDL contains phospholipids with platelet-activating factor-like activity and stimulates the growth of smooth muscle cells. J. Clin. Invest. 96:2322-2330.

29. Stremler, K.E., D.M. Stafforini, S.M. Prescott, and T.M. McIntyre. 1991. Human plasma platelet-activating factor acetylhydrolase: oxidatively-fragmented phospholipids as substrates. J. Biol. Chem. 266:11095-11103.

30. Salari, H. 1986. Solid-phase extraction and reversed-phase high-performance liquid chromatographic technique for isolation and estimation of platelet activating factor in plasma. J. Chromatogr. 382:89-98.

31. Bligh, E.G., and W.J. Dyer. 1959. A rapid method of total lipid extraction and purification. Can. J. Biochem. Physiol. 37:911-917.

32. Weyrich, A.S., M.R. Elstad, R.P. McEver, T.M. McIntyre, K.L. Moore, J.H. Morrissey, S.M. Prescott, and G.A. Zimmerman. 1996. Activated platelets signal chemokine synthesis by human monocytes. J. Clin. Invest. 97:1525-1534.

33. Ikegami, K., C.J. Meade, H.O. Heuer, and F. Birke. 1992. Hetrazepine PAF antagonists. J. Lipid Med. 5:177-182.

34. Lehr, H.A., C. Hubner, B. Finckh, S. Angermuller, D. Nolte, U. Beisiegel, A. Kohlschutter, and K. Messmer. 1991. Role of leukotrienes in leukocyte adhesion following systemic administration of oxidatively modified human low density lipoprotein in hamsters. J. Clin. Invest. 88:9-14.

35. Lehr, H.A., A. Guhlmann, D. Nolte, D. Keppler, and K. Messmer. 1991 Leukotrienes as mediators in ischemia-reperfusion injury in a microcirculation model in the hamster. J. Clin. Invest. 87:2036-2041.

36. Lehr, H.A., J. Seemuller, C. Hubner, M.D. Menger, and K. Messmer. 1993. Oxidized LDL-induced leukocyte/endothelium interaction in vivo in volves the receptor for platelet-activating factor. Arterioscler. Thromb. 13: 1013-1018.

37. Lehr, H.A., B. Frei, A.M. Olofsson, T.E. Carew, and K.E. Arfors. 1995. Protection from oxidized LDL-induced leukocyte adhesion to microvascular and macrovascular endothelium in vivo by vitamin $\mathrm{C}$ but not by vitamin E. Circulation 91:1525-1532.

38. Zimmerman, G.A., S.M. Prescott, and T.M. McIntyre. 1995. Oxidatively fragmented phospholipids as inflammatory mediators: the dark side of polyunsaturated lipids. J. Nutr. 125:S1661-S1665.

39. Weyrich, A.S., T.M. McIntyre, R.P. McEver, S.M. Prescott, and G.A. Zimmerman. 1995. Monocyte tethering by P-selectin regulates monocyte chemotactic protein-1 and tumor necrosis factor- $\alpha$ secretion: signal integration and NF-кB translocation. J. Clin. Invest. 95:2297-2303.

40. Halliwell, B., M. Wasil, and M. Grootveld. 1987. Biologically significant scavenging of the myeloperoxidase-derived oxidant hypochlorous acid by ascorbic acid. FEBS Lett. 213:15-18.

41. Hemila, H., P. Roberts, and M. Wikstrom. 1984. Activated polymorphonuclear leukocytes consume vitamin C. FEBS Lett. 178:25-30.

42. Stremler, K.E., D.M. Stafforini, S.M. Prescott, G.A. Zimmerman, and T.M. McIntyre. 1989. An oxidized derivative of phosphatidylcholine is a substrate for the platelet-activating factor acetylhydrolase from human plasma. $J$. Biol. Chem. 264:5331-5334.

43. Miyaura, S., H. Eguchi, and J.M. Johnston. 1992. Effect of a cigarette smoke extract on the metabolism of the proinflammatory autocoid, plateletactivating factor. Circ. Res. 70:341-347.

44. Tokumura, A., K. Kamiyasu, K. Takauchi, and H. Tsukatani. 1987. Evidence for existence of various homologues and analogs of platelet activating factor in a lipid extract of bovine brain. Biochem. Biophys. Res. Commun. 145 415-425.

45. Kontos, H.A. 1992. Vascular diseases of the limbs. In Cecil Textbook of Medicine. J.B. Wyngaarden, L.H. Smith, and J.C. Bennett, editors. W.B. Saunders Co., Philadelphia. 362.

46. Gey, K.F., U.K. Moser, P. Jordan, H.B. Stahelin, M. Eichholzer, and E. Ludin. 1993. Increased risk of cardiovascular disease at suboptimal plasma concentrations of essential antioxidants: an epidemiological update with special attention to carotene and vitamin C. Am. J. Clin. Nutr. 57:787-797.

47. Eiserich, J.P., A. van der Vliet, G.J. Handelman, B. Halliwell, and C.E. Cross. 1995. Dietary antioxidants and cigarette smoke-induced biomolecular damage: a complex interaction. Am. J. Clin. Nutr. 62:1490-1500. 